\title{
Genomic imprinting: mom and dad (epi)genetics
}

Genomic imprinting is an epigenetic chromosomal modification in the gametes or zygotes that results in a non-random monoallelic expression of specific autosomal genes depending upon their parent of origin. A gene could be maternally imprinted (only paternal copy expressed) or paternally imprinted (only maternal copy expressed). The exact mechanism of genomic imprinting is not clear. However, allele-specific methylation of $\mathrm{CpG}$ residues at the $5^{\prime}$ end of imprinted genes is thought to be responsible for the maintenance of imprinting in somatic cells.

Several lines of evidence have pointed to the presence of imprinted genes in humans and other mammals. Manipulated mouse embryos containing normal diploid chromosome numbers only from the father or only from the mother fail to develop. Human concepti containing two normal sets of genomes $(2 n=46)$ only from the father fail to develop into embryos and instead produce hydatidiform moles. Human triploid abortuses are phenotypically different depending upon whether the extra copy of the genome is from the father or the mother. The first genetic evidence of genomic imprinting came from elegant breeding experiments in the mouse using chromosomal rearrangements to produce uniparental disomy (UPD) for each chromosome and to assess phenotypic effects on embryonic and early development (Cattanach and Kirk 1985). These experiments identified imprinting effects for ten regions of six mouse chromosomes (see Ledbetter and Engel 1995). Deletion of certain specific chromosome regions in humans produces altogether different phenotypes depending upon whether the deletion is on the maternal chromosome or the paternal chromosome. For example, deletion of the segment 15q11-13 on the paternal chromosome produces Prader-Willi syndrome (small hands and feet, mild mental retardation, hypotonia, obesity, and hypogenitalism). The same deletion on the maternal chromosome produces Angelman syndrome (severe mental retardation, seizures, hyperactivity and inappropriate laughter). Certain human genetic traits that are autosomal dominant manifest only when inherited from one parent. For example, BeckwithWiedemann syndrome (gigantism, omphalocele, macroglossia and hemihypertrophy) is expressed only by people who inherit the mutation from their mother. It was not until 1991 that the presence of imprinted genes was reported for the first time in the mouse genome through demonstration of the selective maternal expression of Igf2r and $\mathrm{H} 19$, and the paternal expression of Igf2 (see Morison and Reeve 1998). Since then more than 20 imprinted genes have been identified in mice and humans. In general, imprinted genes are found in a cluster, with the genes imprinted in opposite directions. In the imprinted cluster on human chromosome 11p15.5, H19, KvLQT1, IMPT1, HASH2, p57KIP2 and IPL are maternally expressed whereas IGF2 is paternally expressed. Imprinted genes are known to show tissue-specific imprinting. KvLQT1 is imprinted in all tissues examined so far except in the heart where it shows a biallelic expression. Therefore, the observation of monoallelic expression of a gene in some tissues does not mean that the gene is imprinted in all tissues. Moreover, a gene could be imprinted in one species and not in another. For example, the mouse gene Igf $2 \mathrm{r}$ is paternally imprinted whereas its human homologue IGF2R shows biallelic expression. It is possible that the human gene IGF2R is imprinted in some tissues which have not yet been examined.

Genomic imprinting is reversible and the imprinted status is reset during gametogenesis. For example, in a male who receives an imprinted (inactive) allele of a gene from his father and an expressed allele of the same gene from his mother, the imprinting will be reset for both his alleles during spermatogenesis. In this case, when he passes either allele to his children, both alleles will be imprinted. Similarly, in the case of a female who receives an imprinted allele of the same gene from her father and an expressed allele from her mother, the imprinting process will be reset during her oogenesis so that when she passes either allele to her children, they will be expressed. Thus for a paternally imprinted gene, each individual will have one imprinted allele coming from the father and one expressed allele from the mother. In a similar fashion, the imprinted status of a maternally imprinted gene will be reset during gametogenesis of the mother and the father so that all of their children will receive one imprinted allele from the mother and one expressed allele from the father. 
Uniparental disomy (UPD), in which both members of a chromosome pair come from only one parent, can unmask genomic imprinting and help to identify chromosomes or chromosome segments with imprinted regions. An abnormal phenotype in cases with UPD could result from UPD per se or from the homozygosity of a particular mutant allele present on both copies of chromosome involved in UPD. Although it would be difficult to decide which one is true, an imprinting effect is taken to be certain when three or more cases of UPD for a particular chromosome or chromosome segment produce a similar constellation of abnormal phenotypes (Ledbetter and Engel 1995). It should be noted that autosomal recessive effects are irrespective of imprinting. Based on known UPD cases with abnormal phenotypes, Ledbetter and Engel (1995) have constructed a preliminary imprinted map of the human genome. An imprinting effect is certain for four regions of the human genome: maternal copy of chromosome $7 \mathrm{q}$, paternal copy of chromosome $11 \mathrm{p}$, maternal copy of chromosome $14 \mathrm{q}$, and maternal and paternal copies of the proximal chromosome $15 \mathrm{q}$ (Ledbetter and Engel 1995). Other possible regions of the human genome with imprinting effects are maternal chromosome 2, paternal chromosome 6 , paternal chromosome 14 , maternal chromosome 16 and paternal chromosome 20 (Ledbetter and Engel 1995). So far, imprinted genes from the proximal chromosome 15q (ZNF127, FNZ127, NDN, SNRPN, PAR-SN, PAR5, IPW, PAR1, UBE3A, GABAA), chromosome 11 p (H19, IGF2, HASH2, KvLQT1, p57KIP2, IPL, IMPT1, WT1), chromosome 6q (MAS), chromosome 20 (GNAS), chromosome 7q (MEST), and chromosome 2 (N-MYC) have been reported.

The biological function of genomic imprinting is the subject of considerable debate and remains elusive. More than thirteen theories have been put forth to explain the biological and evolutionary significance of genomic imprinting (see Hurst and McVean 1997). According to the most popular theory - the 'genome conflict' theory-genomic imprinting in placental mammals is the result of an evolutionary conflict between two sets of selfish genomes. Under conditions in which the paternity of future offspring from the same mother is not assured, the paternal genome would favour the removal of nutrients from the mother to the embryo (until such removal endangers the fitness of the embryo). On the other hand, irrespective of who the father is, all offspring born to a mother share their genes with her to the same extent. Therefore the maternal genome would tend to suppress such removal, preferring to keep more of the nutrients in store for offspring yet to be born. Thus the conflict theory predicts that the expression of paternal genes in embryos tends to increase offspring size, whereas the expression of maternal genes tends to reduce it (see Hurst and McVean 1997). Paternal UPD11 cases (Beckwith-Wiedemann syndrome patients) with large size, and maternal UPD7 and maternal UPD14 cases with growth retardation, support this theory. Paternal UPD6 cases with growth retardation on the other hand fail to support the theory.

Genomic imprinting has received a great deal of attention in the past few years due to its role in several human genetic disorders such as Prader-Willi syndrome, Angelman syndrome, Beckwith-Wiedemann syndrome, gestational trophoblastic diseases and cancer. An imprinted gene, IPL, has recently been isolated from human chromosome $11 \mathrm{p} 15.5$ with similarity to TDAG51 which has been implicated in Fas expression and apoptosis (Qian et al 1997). This suggests that genomic imprinting may also have a role in apoptosis. Perhaps the most striking observation concerning the consequences of imprinting has recently been made by Lefebvre et al (1998) regarding the role of the imprinted gene Mest (Peg1) in mice. This gene is expressed only from the paternal allele. Lefebvre et al (1998) introduced a mutation in Mest by gene targeting in embryonic stem (ES) cells and obtained Mest-deficient mice. The loss of Mest function not only resulted in intrauterine growth retardation (IUGR), increased prenatal and postnatal lethality and decreased reproductive fitness of females, but also abnormal maternal behaviour. Normal maternal behaviour in mice includes responding appropriately to newborns, ingesting their extraembryonic tissues after parturition (placentophagia), and influences the time required to retrieve one of the three pups as well as the latency to initiate the construction of a nest with wood chips. Most or all pups born to Mest-deficient female mice were left untouched after parturition, with almost complete absence of placentophagia. Where wild-type females displayed an immediate inclination for nest building, the Mest-deficient female mice performed poorly. Wild-type female mice retrieved the first pup in $\sim 10$ min whereas Mest-deficient female mice did not show this behaviour within the $15 \mathrm{~min}$ period of observation (Lefebvre et al 1998). 
The human homologue of the mouse Mest gene is located on chromosome $7 q 32$. MEST is paternally expressed in human fetal tissues, but biallelically expressed in adult lymphocytes. Maternal UPD7 cases in humans demonstrate intrauterine and postnatal growth retardation. This suggests that the phenotypic consequences of the loss of MEST function are similar in humans and mice with regard to intrauterine and postnatal growth retardation. Thus Lefebvre et al (1998), with their elegant experiment in mice using the paternally expressed gene Mest, have shown for the first time the role of genomic imprinting in animal behaviour. As more imprinted genes are isolated from humans and other mammals, and their roles elucidated, one hopes that the biological purpose of genomic imprinting will not remain elusive for long.

\section{References}

Cattanach B M and Kirk M 1985 Differential activity of maternally and paternally derived chromosome regions in mice; Nature (London) 315 496-498

Hurst L D and McVean G T 1997 Growth effects of uniparental disomies and the conflict theory of genomic imprinting; Trends Genet. 13 436-443

Ledbetter D H and Engel E 1995 Uniparental disomy in humans: development of an imprinted map and its implications for prenatal diagnosis; Hum. Mol. Genet. 4 1757-1764

Lefebvre L, Viville S, Barton S C. Ishino F, Keverne E B and Surani M A 1998 Abnormal maternal behavior and growth retardation associated with loss of the imprinted gene Mest; Nature Genet. 20 163-169

Morison I M and Reeve A E 1998 A catalogue of imprinted genes and parent-of-origin effects in humans and animals; Hum. Mol. Genet. 7 1599-1609

Qian N, Frank D. O'Keefe D, Dao D, Zhao L, Yuan L, Keating M, Walsh C and Tycko B 1997 The IPL gene on chromosome 11 p15.5 is imprinted in humans and mice and is similar to TDAG51, implicated in Fas expression and apoptosis; Hum. Mol. Genet. 6 2021-2029

Arun Kumar

Department of Molecular Reproduction,

Development and Genetics,

Indian Institute of Science, Bangalore 560 012, India

(Email,-karun@hamsadvani.serc.iisc.ernet.in) 